\title{
On the nature of the variable star UNSW-V-760
}

\author{
C. Koen ${ }^{\star}$ \\ Department of Statistics, University of the Western Cape, Private Bag X17, Bellville, 7535 Cape, South Africa
}

Accepted 2010 September 14. Received 2010 September 13; in original form 2010 March 10

\begin{abstract}
The results of multicolour photometric monitoring over several nights of the probable X-ray source UNSW-V-760 are presented. Drawing on both present and past observations, it is shown that the star is multiperiodic and that all the variability can probably be explained by two non-sinusoidal periodicities with periods of 0.276 and $0.198 \mathrm{~d}$. Possible causes (binarity, rotation and oscillation) of the variations are discussed and it is concluded that pulsation must play a role. A classification in the range K1Vke-K3Vke is derived from two low-dispersion spectra. Curiously, the pulsation periods are closer to what is expected for a $\mathrm{K}$ giant star, but the amplitudes are two orders of magnitude larger than those seen in K giants.
\end{abstract}

Key words: stars: individual: UNSW-V-760 - stars: variables: general.

\section{INTRODUCTION}

The subject of this paper is a new variable star discovered in the course of the University of New South Wales (UNSW) photometric search for extrasolar planets (Christiansen et al. 2008). The star is located at RA $07^{\mathrm{h}} 51^{\mathrm{m}} 49.4$, Dec. $-68^{\circ} 14^{\prime} 04^{\prime \prime} .3$ (J2000), and designated UNSW-V-760; this will be abbreviated to 'V760' in the following text. This new variable has a position very close to the ROSAT detection 2RXP J075145.0-681416 - offset 4 arcsec, with a positional uncertainty of 13 arcsec (Voges et al. 2000). It is therefore a probable X-ray emitter. It is noted in passing that there is also an Einstein detection, 2E 1887, 17 arcsec from the position of V760 (positional uncertainty 52 arcsec) (Moran et al. 1996). Given the sparseness of the field, it seems likely to be the same source.

The UNSW observations show the star to be a small amplitude (26 mmag) periodic $(P=0.20 \mathrm{~d})$ variable and it was assigned the type 'PUL' (i.e. a pulsating variable). The UNSW observations were obtained through a Johnson $I$ filter, the mean brightness being $10.70 \mathrm{mag}$. There is no published spectral type.

The combination of properties of the star is interesting. Such short pulsation periods are usually associated with $\delta$ Scuti stars; these are dwarfs or giants of spectral types roughly A2-F3. However, such early-type stars are not usually X-ray sources. On the other hand, there are later-type variable stars (BY Draconis stars), which show sinusoidal light variations due to the presence of dark spots on their surfaces. The periodic light modulations seen in these stars are due to stellar rotation, which could be quite rapid. Typical spectral classifications are $\mathrm{dKe}$ or $\mathrm{dMe}$, indicating chromospheric activity and hence the possibility of X-ray radiation. A further possibility, which could explain the observational facts, is that the star is a contact binary (W UMa star): light curves of many of these variables are close to sinusoidal. For the period to be as short as $0.2 \mathrm{~d}$, the

${ }^{\star}$ E-mail: ckoen@uwc.ac.za components would have to be physically small, implying very late spectral types, with the concomitant common property of being magnetically active.

The work reported in this paper was stimulated by an attempt to discover the precise nature of V760. Photometric observations are described in Section 2. Section 3 contains an analysis of the UNSW photometry. Section 4 contains an analysis of the author's own time series photometry. A spectroscopic classification is derived in Section 5. The interpretation of all the available data is discussed in Section 6 and the paper concluded in Section 7.

\section{PHOTOMETRIC OBSERVATIONS AND REDUCTIONS}

Observations were made with the South African Astronomical Observatory (SAAO) CCD cameras mounted on the SAAO 1.0-m telescope. Measurements during the first two nights were made with the STE4 camera, with a field of view of about $5 \times 5 \mathrm{arcmin}^{2}$; thereafter, the STE3 camera with a smaller $\left(\sim 2.5 \times 2.5 \mathrm{arcmin}^{2}\right)$ field of view was used. Both cameras have SITe back-illuminated chips. Prebinning $(2 \times 2)$ was used throughout, giving readout times of roughly 20 and $5 \mathrm{~s}$ for the two cameras, respectively.

On one night, measurements were made through a $U$ filter only; on five nights, observations were made through a $B$ filter; and on the remaining three nights, contemporaneous observations were obtained in $B V R_{\mathrm{C}} I_{\mathrm{C}}$, by cycling continuously through the four filters. A $\log$ of the observations is given in Table 1. In what follows, the subscript ' $\mathrm{C}$ ' on $R$ and $I$ will be dropped for convenience. Exposure times were tailored to observing conditions, which varied from excellent (photometric, with seeing of 1 arcsec or better) to poor (cirrus and/or seeing of several arcsec).

Reductions were obtained online by an automated version of Dорнот (Schechter, Mateo \& Saha 1993). Magnitudes determined from the fitting of point spread functions were used throughout. 
Table 1. The SAAO photometric observing $\log : T_{\exp }$ is the exposure time and $N$ is the minimum number of useful measurements per filter obtained during a given run. The short exposure times in $I$ were necessitated by the rapid saturation of images in this band.

\begin{tabular}{ccccc}
\hline $\begin{array}{c}\text { Starting time } \\
\text { (HJD 2455190+) }\end{array}$ & Filter & $\begin{array}{c}T_{\exp } \\
(\mathrm{s})\end{array}$ & $\begin{array}{c}\text { Run length } \\
(\mathrm{h})\end{array}$ & $N$ \\
\hline 4.5018 & $B$ & 50 & 2.3 & 122 \\
5.3069 & $B$ & 40 & 6.9 & 428 \\
6.3273 & $B$ & $25-60$ & 2.6 & 133 \\
7.3250 & $B V R I$ & $30,8-12,4-10,4-7$ & 4.2 & 160 \\
8.3042 & $B$ & $30-40$ & 2.3 & 72 \\
9.2985 & $B$ & 40 & 2.1 & 155 \\
10.3073 & $B V R I$ & $30-40,12-15,8-12,5-8$ & 6.5 & 213 \\
11.3355 & $U$ & 180 & 4.3 & 85 \\
12.3376 & $B V R I$ & $30,12,8,5$ & 4.6 & 213 \\
\hline
\end{tabular}

In order to cope with changeable weather conditions, the photometry was differentially corrected using the measurements of two bright stars in the field of view. Typical light curves are shown in Figs 1 and 2. Cycle lengths of roughly $5 \mathrm{~h}$ are clearly visible. Further inspection shows that successive minima do not have the same depths, suggesting that the variability is non-sinusoidal and/or multiperiodic.
The filterwheel was replaced after the first three nights of operation due to technical problems. This entailed using a different set of filters, which - at least in theory - is equivalent to the first set. However, the mean differential $B$ magnitude was 0.07 mag brighter after the change. Since only the $B$ filter was used for the first three runs, no statement can be made about the remaining filters. Since the two local standard stars have colours indicative of spectral class $\mathrm{G}$, and V760 is a K star (see below), the colours are not very different and this change in relative brightnesses is therefore difficult to understand. The filters were examined and found to be clean and without obvious signs of damage. The possibility of a real change in the mean brightness of V760 cannot be excluded - otherwise, the author is at a loss for an explanation. If the second filter set was defective in some way, then it may have affected the absolute photometry reported below - but it should be said that there are no overt indications of problems with the photometry.

It is worth remarking on the absence of any obvious flares in the photometry. This suggests that the X-ray emission in V760 is steady or that the star is not very active.

There is no published spectral classification for V760. An effort was therefore in the first instance made to obtain standardized photometric indices. To this end, three bright late-type (G8-K0) standard stars were each measured several times in BVRI under good photometric conditions. The observations were corrected for

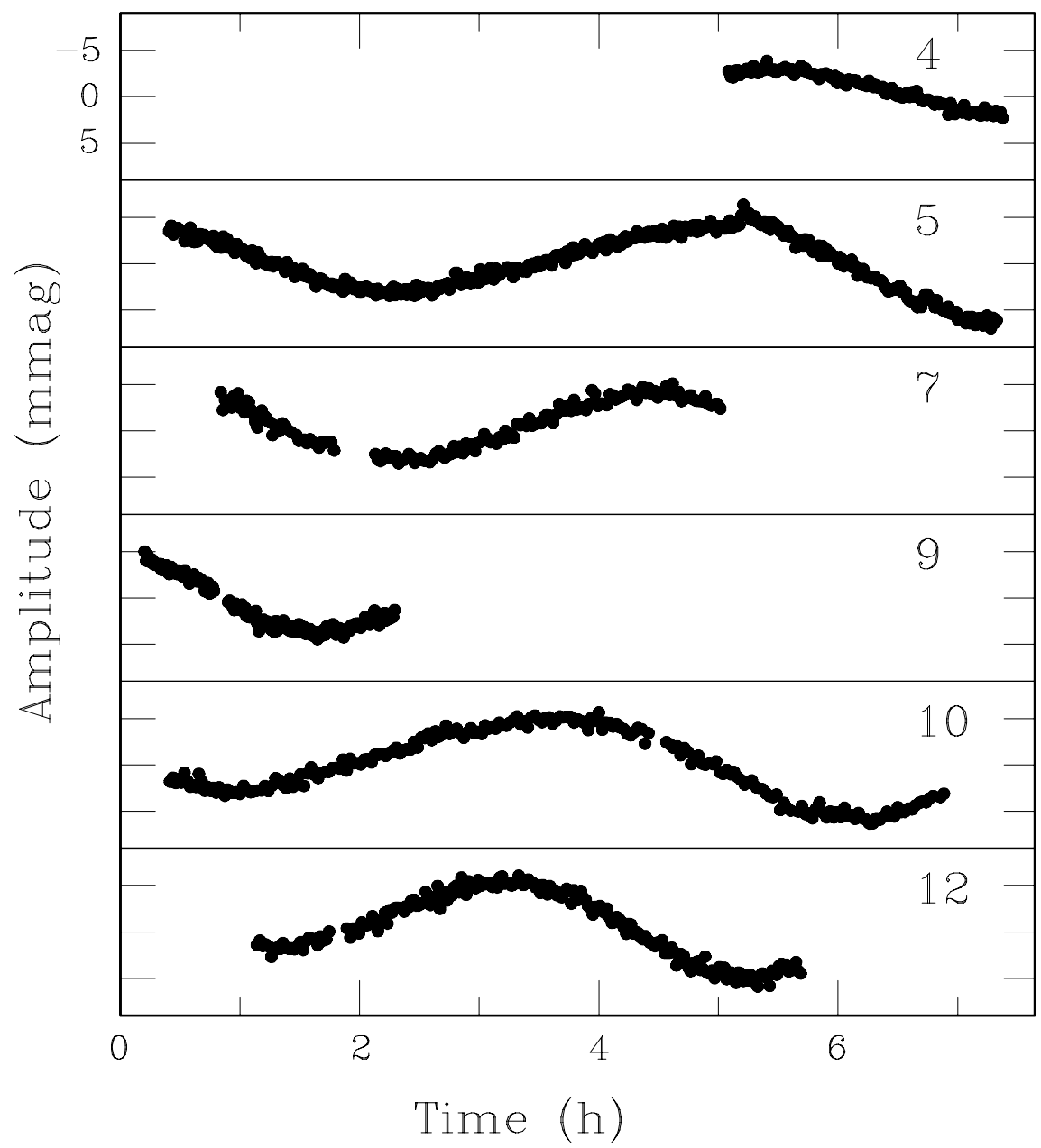

Figure 1. The bulk of the differentially corrected $B$-filter observations. The different panels are labelled with the date in the form of days since JD 2455190 . An offset of $0.07 \mathrm{mag}$ has been subtracted from measurements for the first two nights to bring these in line with the rest of the data (see text for details). The height of each of the panels is $0.18 \mathrm{mag}$. 


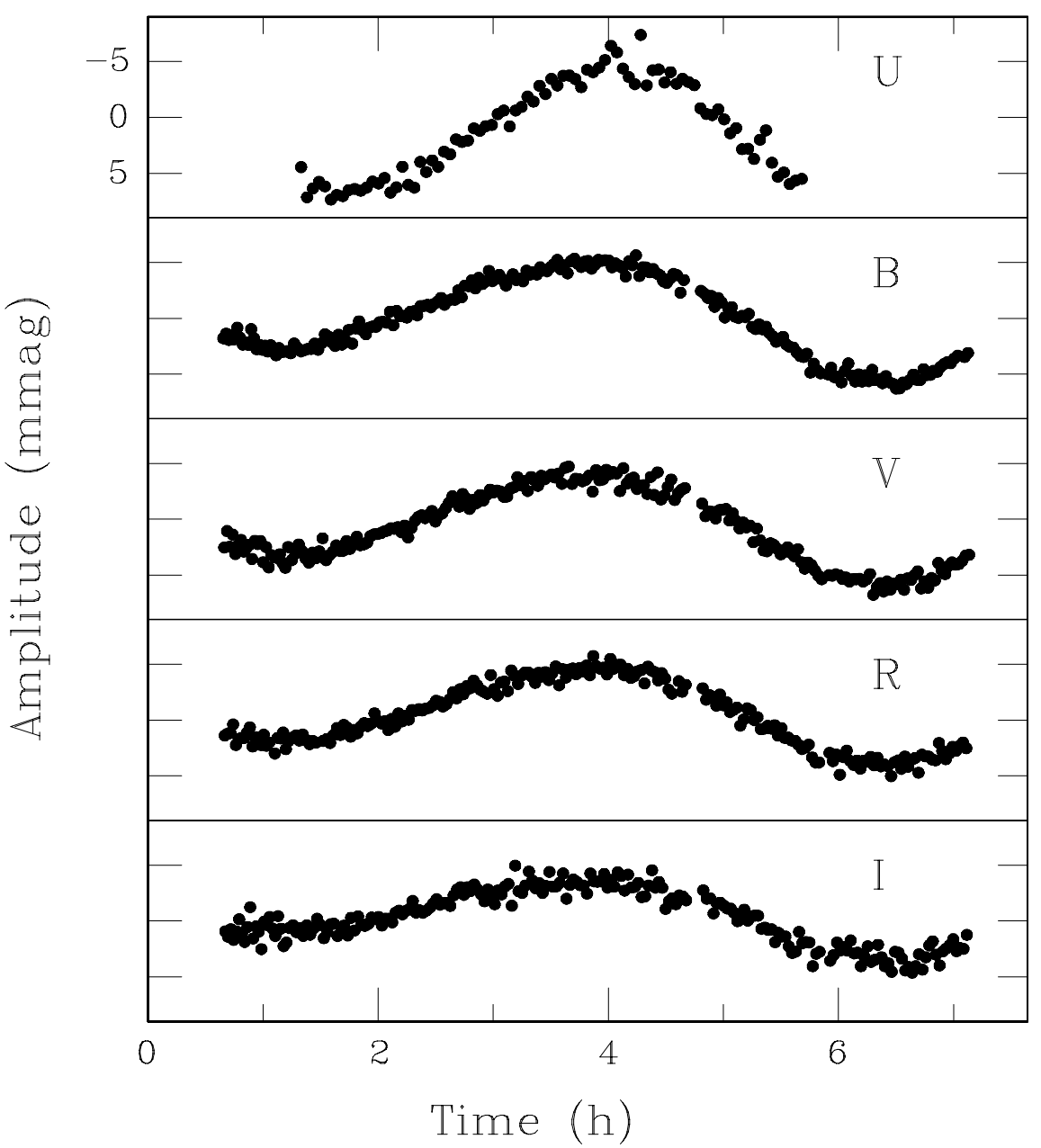

Figure 2. A comparison of the observations obtained through the different filters. Measurements in BVRI were obtained contemporaneously (on JD 2455200 ), while the $U$-filter observations were made the next night. The height of each of the panels is 0.18 mag.

the first-order airmass effect, using standard extinction coefficients. No higher order corrections were made, as colour equations were not available. This deficiency should not be too important, since, as is shown below, V760 is of similar type to the standards. Mean photometry of the three standard stars was used to set the photometric zero-points directly before commencing observations of V760. The first 40 measurements of the V760 field, in each of $B V R I$, showed no significant trends and were used to obtain standardized photometry of V760 and two bright local comparison stars in the field of view. The standard deviations of the observations were much the same for the three stars and ranged from $7 \mathrm{mmag}$ in $B$ to $13 \mathrm{mmag}$ in $I$. Results for V760 are $V=12.304,(B-V)=1.020,(V-R)=0.604$ and $(V-I)=1.212$. These colour indices suggest a spectral type of about K2-K5V, or K0-K3III, by comparison with the photometry of standard stars with known spectral types (Menzies et al. 1989).

Standardized colour-corrected photometry of V760 was also obtained with the Modular Photometry attached to the SAAO 0.5-m telescope. Measurements were obtained on three nights: differences of a few hundredths of a magnitude were seen, with the star clearly bluer when brighter. Mean values were $V=12.27,(B-V)=1.06$, $(V-R)=0.62$ and $(V-I)=1.20$. Although individual observations on the $0.5-\mathrm{m}$ telescope are less accurate, these mean results are probably a better reflection of the average values for V760.

The 2MASS photometry of V760 gives $J=10.208, H=9.652$ and $K_{\mathrm{S}}=9.491$ (Skrutskie et al. 2006). Bessell \& Brett (1988) have supplied calibrations of spectral types on Glass (SAAO) nearinfrared indices. The 2MASS indices $(J-H)=0.56$ and $(H-$ $\left.K_{\mathrm{S}}\right)=0.16$ are therefore transformed to $(J-H)=0.64,(H-K)=$ 0.13 in the SAAO system, using the simplified equations of Koen et al. (2007). These infrared photometric indices imply spectral types K5-K7V or K2-K3III.

As is clear from the above, the positions of $\mathrm{K}$ dwarfs and giants overlap in two-colour diagrams. Inspection of the model colours plotted in Bessell, Castelli \& Plez (1998) suggests that the (V $K)$ and $(J-K)$ indices may have the best discriminatory power (see their fig. 10). Again using the equations of Koen et al. (2007), the Glass system indices $(V-K)=2.79$ and $(J-K)=0.76$ are obtained: these slightly favour classification as a giant.

No account has been taken of possible reddening in the above. The reasonable agreement between classifications based on the nearinfrared indices, and those calculated from the optical photometry, indicates that reddening is probably absent or small.

We digress briefly to discuss the difference between the UNSW Johnson $I$ measurement (10.70), the Cousins $I$ measured at SAAO (11.09) and the DENIS I measurement (11.01; Epchtein et al. 1999). The effective wavelengths and 50 per cent transmission intervals of the three filters are compared in Table 2. Information for the Johnson and Cousins systems was extracted from Bessell (1979). The filter used in the DENIS was a 'Gunn' $i$ (Fouqué et al. 2000); the commonly used name seems to be a misnomer, since the filter 
Table 2. A comparison of the three different $I$ filters through which measurements of V760 have been made.

\begin{tabular}{lcc}
\hline System & $\begin{array}{c}\text { Effective wavelength } \\
(\mu \mathrm{m})\end{array}$ & $\begin{array}{c}50 \text { per cent transmission interval } \\
(\mu \mathrm{m})\end{array}$ \\
\hline Johnson & 0.90 & $0.75-0.98$ \\
Cousins & 0.79 & $0.725-0.875$ \\
DENIS & 0.82 & $0.75-0.88$ \\
\hline
\end{tabular}

was apparently first introduced and described in Wade et al. (1979). For a 5000-K blackbody, the wavelength of peak radiation is $0.58 \mu \mathrm{m}$, so that all three filters measure radiation in the red tail of the star's continuum. The relatively small difference between the SAAO (Cousins) and DENIS measurements can probably be ascribed to measurement errors (given as 0.03 for the DENIS observation and at least 0.013 in the SAAO measurement). The bandpass of the Johnson filter is much wider and this may account for the fact that the star is measured $\sim 0.4$ mag brighter in the UNSW survey.

\section{UNIVERSITY OF NEW SOUTH WALES OBSERVATIONS}

Before embarking on analysis of the SAAO data, it is useful to study the UNSW measurements. The UNSW data base has 3004 observations of V760, extending over $101.12 \mathrm{~d}$. The distribution of the individual photometric errors has an extended tail, with a few values larger than 1 mag. Selecting only those observations with errors smaller than 0.025 mag eliminates most questionable measurements, leaving only six high outliers (Fig. 3). These are also discarded in what follows, in order to avoid their possibly excessive influence on frequency spectra - the number of usable observations is then 2972 .

An amplitude spectrum of the data is plotted in the top panel of Fig. 4. The highest peak is at a frequency of $5.05336 \mathrm{~d}^{-1}$. If a sinusoid with this frequency is fitted to the data by least squares, and the fitted sinusoid is subtracted (pre-whitened) from the data, the spectrum of the residuals reaches a maximum at $f=3.6281 \mathrm{~d}^{-1}$ (second panel of Fig. 4). Continuing in this vein, the essentially featureless spectrum in the bottom panel of Fig. 4 is obtained after pre-whitening five frequencies from the observations.

As a check, the same procedure was carried out separately on the two halves of the data. All the results are summarized in Table 3. Frequencies of 5.053, 3.628 and $10.108 \mathrm{~d}^{-1}$ are consistently present. Other frequencies could be ascribed to low-frequency noise, longer term trends in the star's brightness, extinction effects or perhaps slight errors in nightly zero-points.

Finally, we note that $5.05336 \times 2=10.10672$, which suggests that the low-amplitude periodicity with $f \sim 10 \mathrm{~d}^{-1}$ is in fact the first harmonic of the largest amplitude term. Of course, this implies that the $f=5.05336 \mathrm{~d}^{-1}$ periodicity is non-sinusoidal.

\section{ANALYSIS OF THE SAAO OBSERVATIONS}

An amplitude spectrum of the SAAO $B$-filter data can be seen in the top panel of Fig. 5. It is dominated by the peak at $5.053 \mathrm{~d}^{-1}$, together with its aliases. If this feature is pre-whitened from the data, the highest peak in the spectrum of the residuals is at $3.62 \mathrm{~d}^{-1}$. These frequencies were also the most prominent in the UNSW data (Table 3). The highest peak in the third panel of Fig. 5 is at $8.25 \mathrm{~d}^{-1}$; there is no equivalent of this in the UNSW observations. Furthermore, examination of the bottom panel of Fig. 5 shows that there is no feature near $10 \mathrm{~d}^{-1}$ matching the $10.11 \mathrm{~d}^{-1}$ frequency in the UNSW data.

In order to proceed, it is assumed that the first two frequencies in the SAAO data are exactly equal to the (better-determined) first two frequencies in Table 3. These are then pre-whitened from all the SAAO observations. Spectra of the residuals are plotted in Fig. 6. There are low-frequency power excesses in $V$ and $R$, which are ignored for the time being. Aside from these, all the amplitude spectra reach maxima near $7.5 \mathrm{~d}^{-1}$ (or $1 \mathrm{~d}^{-1}$ aliases): the precise frequencies are $7.26(B), 6.26(V), 7.23(R)$ and $7.20 \mathrm{~d}^{-1}(I)$. It seems reasonable to assume that these are all due to the same mechanism and that the intrinsic (as opposed to the observed) frequencies are the same in all four cases. Since the $B$-filter data are the most extensive, it is assumed that the correct frequency is $7.265 \mathrm{~d}^{-1}$. Table 4 contains the results of fitting the three frequencies 5.05336 , 3.62813 and $7.26465 \mathrm{~d}^{-1}$ to the SAAO data.

If the three frequencies in Table 4 are pre-whitened from each of the $B V R I$ data sets, the residual spectra in Fig. 7 follow. There is considerable low-frequency $\left(f<4 \mathrm{~d}^{-1}\right)$ power in the residuals. It is not clear how much of this is due to imperfect matching of the data from various nights (e.g. the zero-point shift after the first three nights) and how much is attributable to true variability in V760. There is little noteworthy at higher frequencies. However, if the pre-whitening process is continued, Fig. 8 is obtained. (A further two frequencies have been pre-whitened from the $B V R$ data and one from the $I$-filter observations.) Remarkably, the highest peaks are all near the third of the UNSW frequencies $\left(10.1083 \mathrm{~d}^{-1}\right.$ - see Table 3$)$ or its aliases: $11.105(B), 11.06(V), 10.53(R)$ and $10.10 \mathrm{~d}^{-1}(I)$. The reader may object that the frequency $10.53 \mathrm{~d}^{-1}$ extracted from

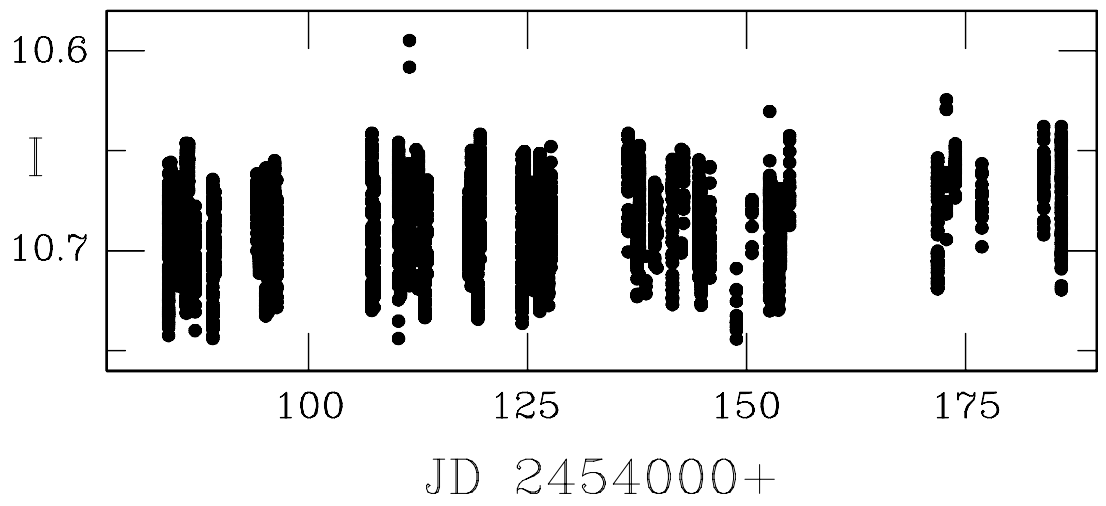

Figure 3. The UNSW observations of V760. A total of 26 measurements with photometric errors in excess of 0.025 mag have been excluded. 


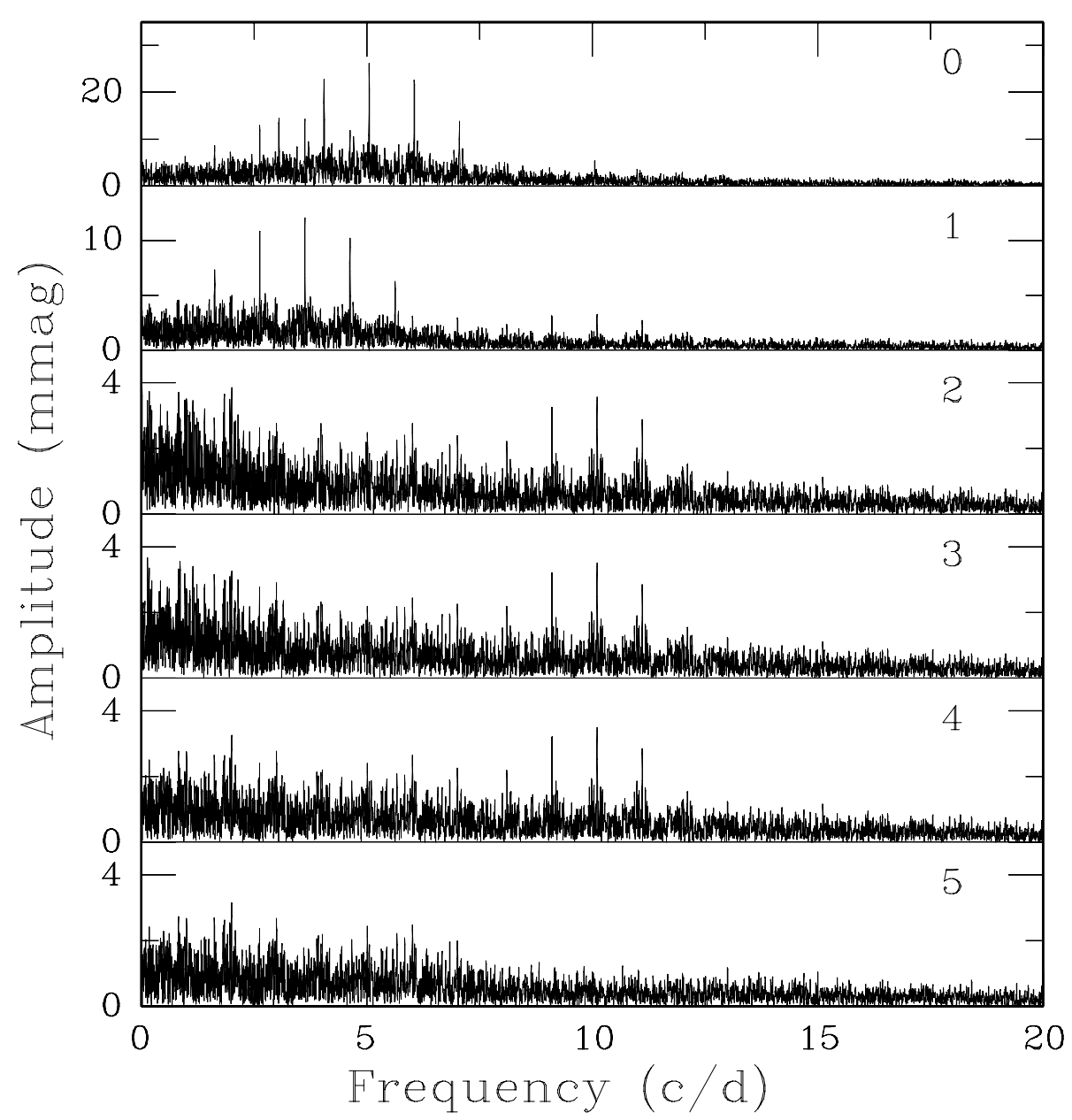

Figure 4. A frequency spectrum of the UNSW observations of V760 and spectra of the residuals after several stages of pre-whitening. Each of the panels is labelled with the number of fitted sinusoids, which have been subtracted from the original data set. Note the different scales on the different vertical axes.

Table 3. The results of successive pre-whitening of the UNSW data by sinusoids. The first two columns give results for the full data set and the remainder column gives results for the first and second halves of the data. The formal uncertainty in the last digit of an estimate is given in brackets.

\begin{tabular}{cccccc}
\hline \multicolumn{2}{c}{ All data } & \multicolumn{2}{c}{ First half } & \multicolumn{2}{c}{ Second half } \\
$\begin{array}{c}\text { Frequency } \\
\left(\mathrm{d}^{-1}\right)\end{array}$ & $\begin{array}{c}\text { Amplitude } \\
(\mathrm{mmag})\end{array}$ & $\begin{array}{c}\text { Frequency } \\
\left(\mathrm{d}^{-1}\right)\end{array}$ & $\begin{array}{c}\text { Amplitude } \\
(\mathrm{mmag})\end{array}$ & $\begin{array}{c}\text { Frequency } \\
\left(\mathrm{d}^{-1}\right)\end{array}$ & $\begin{array}{c}\text { Amplitude } \\
(\mathrm{mmag})\end{array}$ \\
\hline $5.05336(7)$ & $26.8(3)$ & $5.0538(3)$ & $27.9(5)$ & $5.0539(2)$ & $25.8(5)$ \\
$3.6281(1)$ & $12.2(2)$ & $3.6287(4)$ & $13.1(3)$ & $3.6286(3)$ & $10.8(4)$ \\
$0.0158(4)$ & $3.3(2)$ & $0.967(1)$ & $4.2(3)$ & $1.0772(4)$ & $6.2(3)$ \\
$0.1408(3)$ & $3.7(2)$ & $10.107(1)$ & $3.6(3)$ & $0.0170(5)$ & $5.2(4)$ \\
$10.1083(3)$ & $3.5(2)$ & $0.657(1)$ & $3.3(3)$ & $1.4029(7)$ & $3.5(3)$ \\
$2.0045(3)$ & $3.3(3)$ & $6.010(2)$ & $2.4(2)$ & $0.427(1)$ & $2.5(3)$ \\
$5.0130(4)$ & $2.7(2)$ & $1.613(2)$ & $2.1(2)$ & $5.0236(7)$ & $3.1(3)$ \\
$0.8257(5)$ & $2.3(1)$ & $1.460(2)$ & $2.0(3)$ & $10.1099(8)$ & $2.6(3)$ \\
\hline
\end{tabular}

the $R$-filter observations is not at a $1 \mathrm{~d}^{-1}$ alias, but a glance at the window function of these data (Fig. 9) shows substantial aliasing also near 0.4 and $0.6 \mathrm{~d}^{-1}$.

Fig. 8 implies that the $10.1083 \mathrm{~d}^{-1}$ frequency found in the UNSW data is indeed present in the SAAO data, albeit at very low levels. The logical next step is to fit all three UNSW frequencies, plus $f=$ $7.265 \mathrm{~d}^{-1}$, to the SAAO data. However, it was pointed out at the end of Section 3 that the main periodicity is probably non-sinusoidal, that is, the first and third UNSW frequencies are not independent. We now note that $3.6281 \times 2=7.2562$, which is very close to the new frequency found in the SAAO data. Table 5 shows the results of fitting the two independent frequencies found in the UNSW data, and their first harmonics, to the SAAO data.

The large phase difference between the $3.628 \mathrm{~d}^{-1}$ variation in $B$ and in the other three filters is strange. There is also a large phase difference between its harmonic as measured in $B$ versus the other 


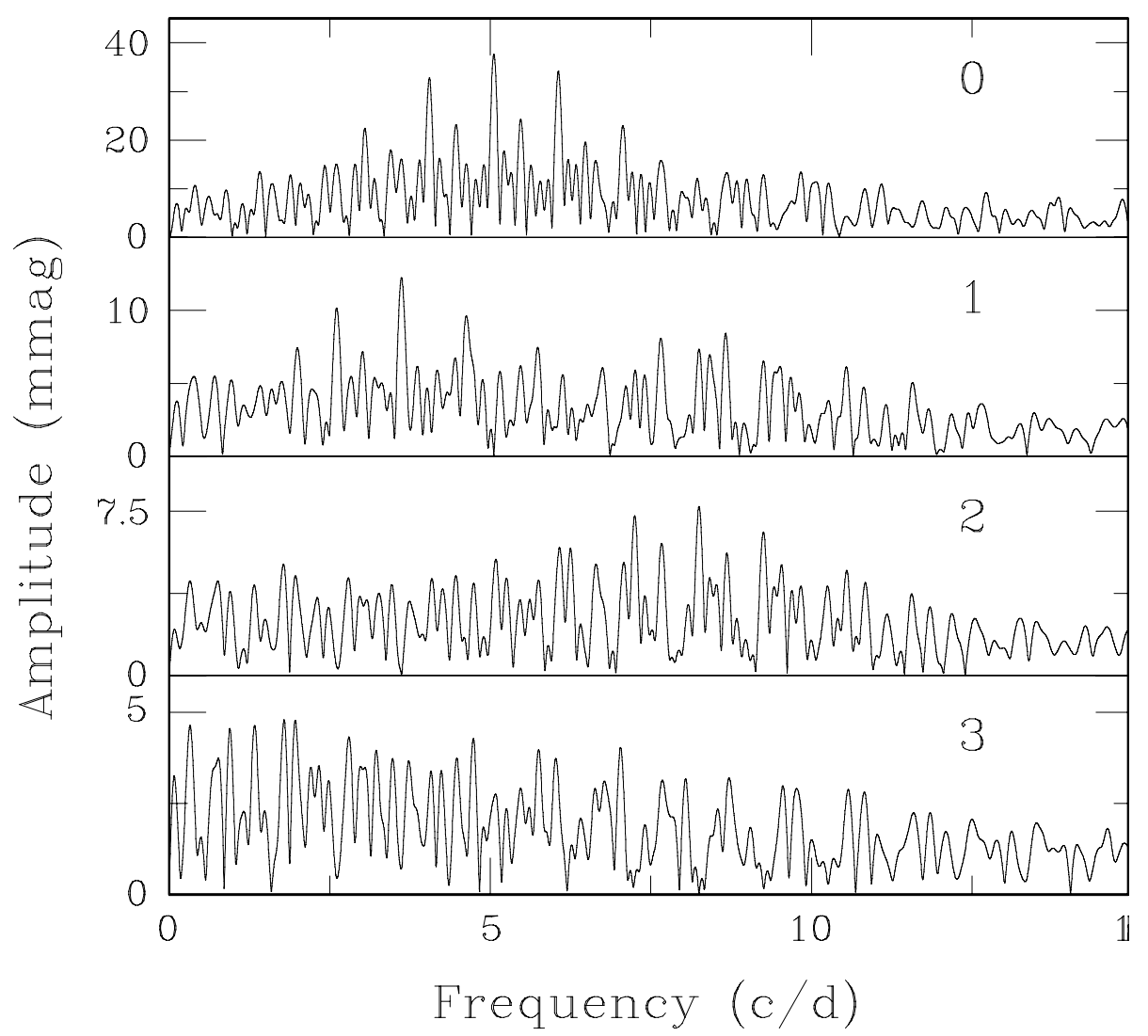

Figure 5. As for Fig. 4, but applied to the SAAO $B$-filter data.

three filters. Excluding the first three nights' $B$-filter data made some difference: the phase associated with $f=3.628 \mathrm{~d}^{-1}$ changed to 0.721 (formal standard error 0.019), which is closer to, but still markedly different from the results for the other filters. Interestingly, the phase of $f=7.2562=2 \times 3.628 \mathrm{~d}^{-1}$ changed to -0.190 (s.e. 0.046 ) - very similar to the results at the longer wavelengths.

It is noteworthy that the Table 5 I-filter amplitudes of the 5.053 and 3.628 variations are very similar to those found in the UNSW data (Table 3). On the other hand, the $10.1 \mathrm{~d}^{-1}$ variations have rather different amplitudes, while the $7.26 \mathrm{~d}^{-1}$ periodicity is absent in the UNSW observations. The two data sets were obtained about $1000 \mathrm{~d}$ apart: clearly, the shape of the light curve can change on this, or shorter, time-scales. In fact, the phase results discussed in the preceding paragraph suggest that the light curve may evolve on a time-scale of days.

\section{MK CLASSIFICATION}

The standardized photometry discussed in Section 2 establishes V760 as an early to mid K star, but does not allow deduction of the luminosity class. As this is of importance in the interpretation of the results of Sections 3 and 4, details of the MK classification are presented.

Two low-dispersion spectra of V760 were obtained with the CCD Cassegrain spectrograph attached to the SAAO 1.9-m telescope (Table 6). Arc spectra obtained immediately before and after each observation were used to wavelength-calibrate observations. Spectra were also roughly flux-calibrated, using observations of a single spectrophotometric standard observed some hours before V760.
Fig. 10 compares the 2 - $\AA$ resolution spectrum of V760 (middle panel) with similar (1.8- $\AA$ ) resolution spectra of 54 Psc (K0V, top panel); $61 \mathrm{CygA}$ (K5V, second from the top panel); $\eta$ Cyg (K0III, second from the bottom panel); and $\alpha$ Tau (K5III, bottom panel). These bright MK standard star spectra were downloaded from the site http://stellar.phys.appstate.edu/Standards/stdindex.html which provides data supplementing Gray \& Corbally (2009). Fig. 11 compares $1-\AA$ resolution spectra obtained during the same night, and with the same equipment, of the bright K4/5 dwarf HR 8387 (top panel) and V760 (bottom panel). Inspection of the diagrams shows the following:

(i) A comparison of the Sri 4077 line, and the Sr II absorption feature at $\lambda 4215 \AA$, with those of dwarfs and giants shows that V760 has the weak lines of a dwarf. Also note that the $\mathrm{CN}$ band depression, particularly notable in the KOIII spectrum, is absent in the V760 spectrum.

(ii) The absorption depths at $\lambda \lambda 4250$ and $4254 \AA$ are similar, indicating a type earlier than $\mathrm{K} 5$.

(iii) The CrI 4290 and Fe I 4144 lines are markedly weaker in V760 than they are in HR 8387, suggesting a spectral type earlier than $\mathrm{K} 4$.

(iv) The $\mathrm{V} 760 \mathrm{H} \delta$ absorption at $4102 \AA$ is very weak: this is most likely a consequence of chromospheric emission, which is expected on the basis that the star is an X-ray source. Filling in of other Balmer lines is also evident, and emission cores are prominent in the $\mathrm{Ca}$ II $\mathrm{H}$ and $\mathrm{K}$ lines.

Further relevant information is supplied by a flux depression in the region 5000-5200 ̊ and moderately strong Fe I $5207 \AA$ absorption: these features imply a spectral type later than K0 (compare to 


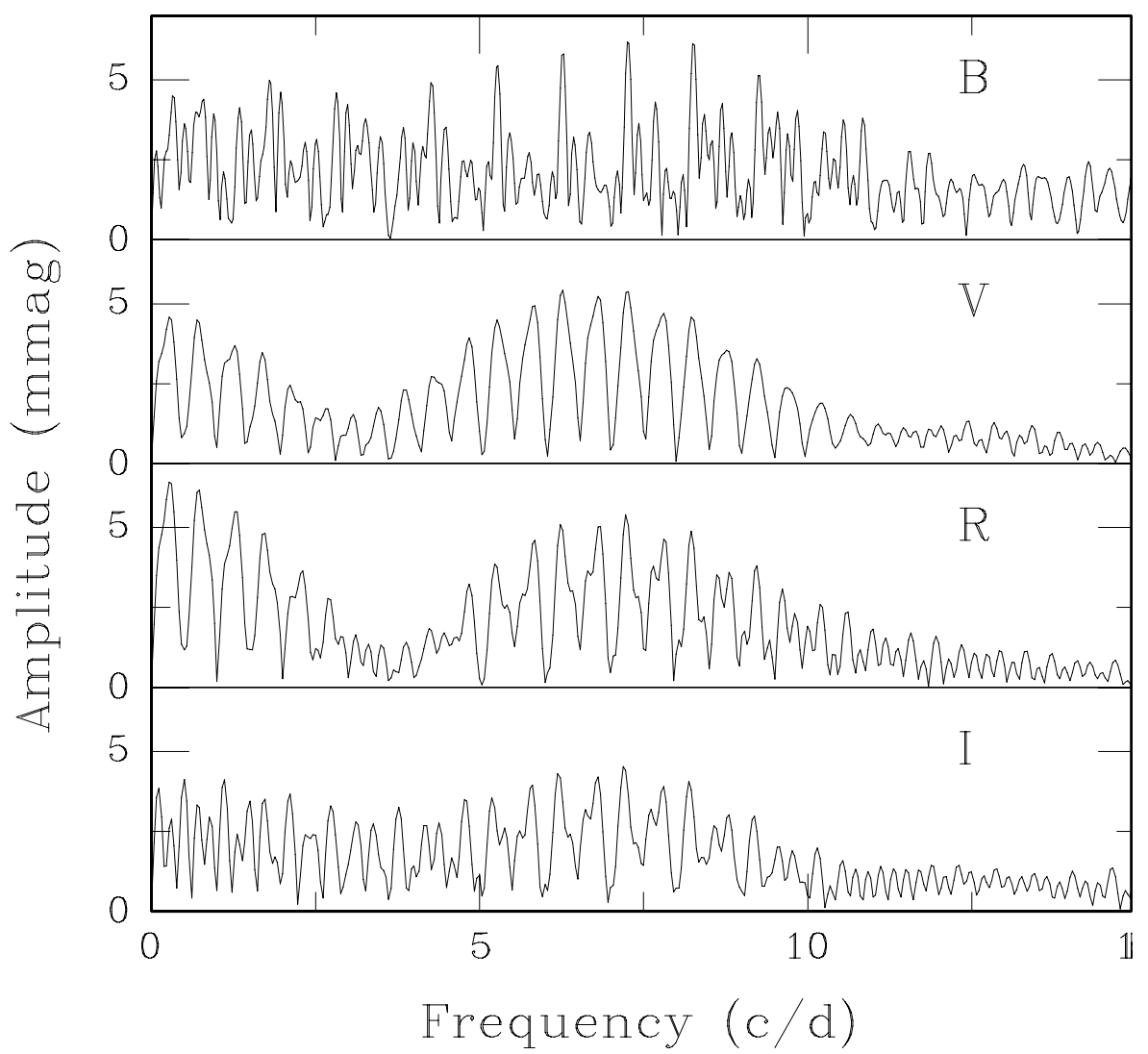

Figure 6. Amplitude spectra after pre-whitening the first two frequencies in Table 3 from each of the four (i.e. BVRI) SAAO data sets. Panels are labelled with the filter used.

Table 4. The results of fitting three frequencies to the SAAO time series data. For each frequency, the first line gives amplitudes and the second line gives phases. The uncertainty in the last digit of an entry is given in brackets.

\begin{tabular}{ccccc}
\hline Frequency $\left(\mathrm{d}^{-1}\right)$ & \multicolumn{4}{c}{ Amplitude (mmag)/phase (rad) } \\
& $B$ & $V$ & $R$ & $I$ \\
\hline \multirow{2}{*}{5.05336} & $41.0(3)$ & $43.8(6)$ & $38.5(6)$ & $29.9(7)$ \\
& $2.842(9)$ & $2.88(1)$ & $2.86(1)$ & $2.82(2)$ \\
3.62813 & $15.3(4)$ & $16.2(6)$ & $15.0(6)$ & $13.6(6)$ \\
& $0.46(2)$ & $0.96(4)$ & $0.96(4)$ & $0.92(5)$ \\
7.265 & $6.7(3)$ & $6.3(4)$ & $6.0(4)$ & $4.3(5)$ \\
& $-0.62(4)$ & $-0.65(6)$ & $-0.66(7)$ & $-0.6(1)$ \\
\hline
\end{tabular}

e.g. fig. 7.2 in Gray \& Corbally 2009). Taken together, a classification of K1Vke-K3Vke, in the nomenclature of Gray \& Corbally (2009), is derived ('ke' indicates moderately strong emission).

\section{DISCUSSION}

There are three possible sources of the periodicities: binarity (ellipsoidal variability), rotation (starspots) and pulsation. These are considered in turn.

Kepler's third law can be written in the form

$\sqrt{\frac{A^{3}}{M_{1}+M_{2}}}=8.64 P$,

where $A$ is the binary separation (in $\mathrm{R}_{\odot}$ ), $M_{1}$ and $M_{2}$ are the component masses (in $\mathrm{M}_{\odot}$ ), and $P$ is the period (in days). For the longer of the two principal periods found in Sections 3 and $4\left(P_{1}=0.276 \mathrm{~d}\right)$,

$\sqrt{\frac{A^{3}}{M_{1}+M_{2}}}=4.77$

or

$A=2.83\left(M_{1}+M_{2}\right)^{1 / 3}$,

where it has been assumed that the binarity will manifest as ellipsoidal variability, and hence the observed period would be twice $P_{1}$. Given that V760 is a dwarf, a late main sequence, or compact companion, could be accommodated. The binary would be very close and rotation of the components would be synchronized with the binary period. At least some of the remaining periodicities would therefore have to be the result of pulsation, rather than rotation.

Equation (1) can also be used to address rotation as a source of periodicity: the breakup (Keplerian) rotation period at the stellar surface is

$P_{\mathrm{b}}=0.12 \sqrt{\frac{R^{3}}{M}}$

where $R$ is the radius and $M$ is the mass. In order that $P_{1}>P_{\mathrm{b}}$,

$R<1.79 M^{1 / 3}$

is required: for a $\mathrm{K}$ giant, the implied rotation velocity would be greater than the breakup value. As the star is a dwarf, it is physically possible that at least one of the periodicities could be due to rotation.

Even shorter rotation periods have been observed in late-type main-sequence stars (e.g. Meibom, Mathieu \& Stassun 2009). It is believed that in $\mathrm{K}$ dwarfs, rotation slows down at ages larger than about $140 \mathrm{Myr}$, implying that V760 would need to be relatively 


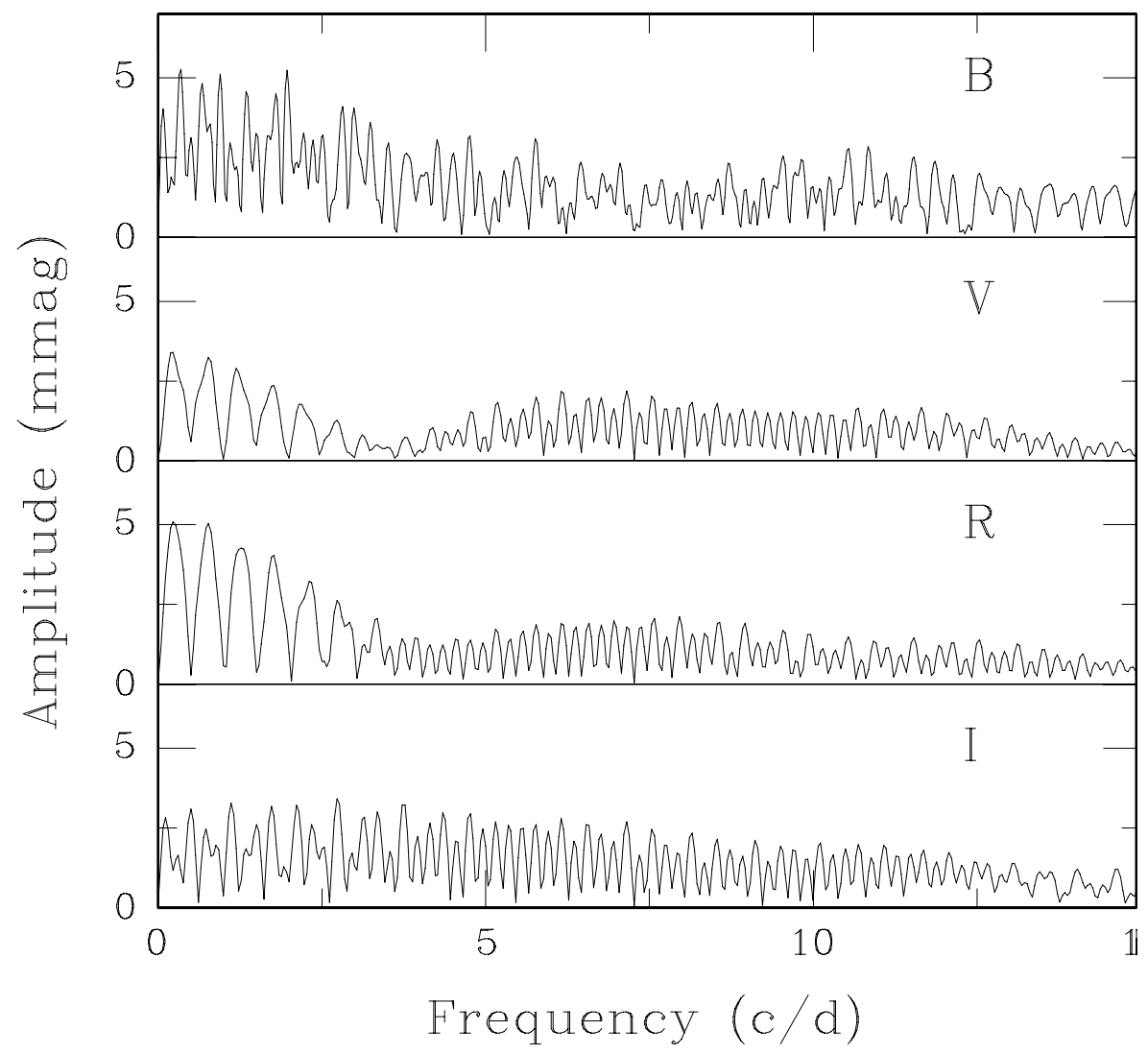

Figure 7. As for Fig. 6, but after pre-whitening by three frequencies $\left(5.05,3.63\right.$ and $\left.7.26 \mathrm{~d}^{-1}\right)$.

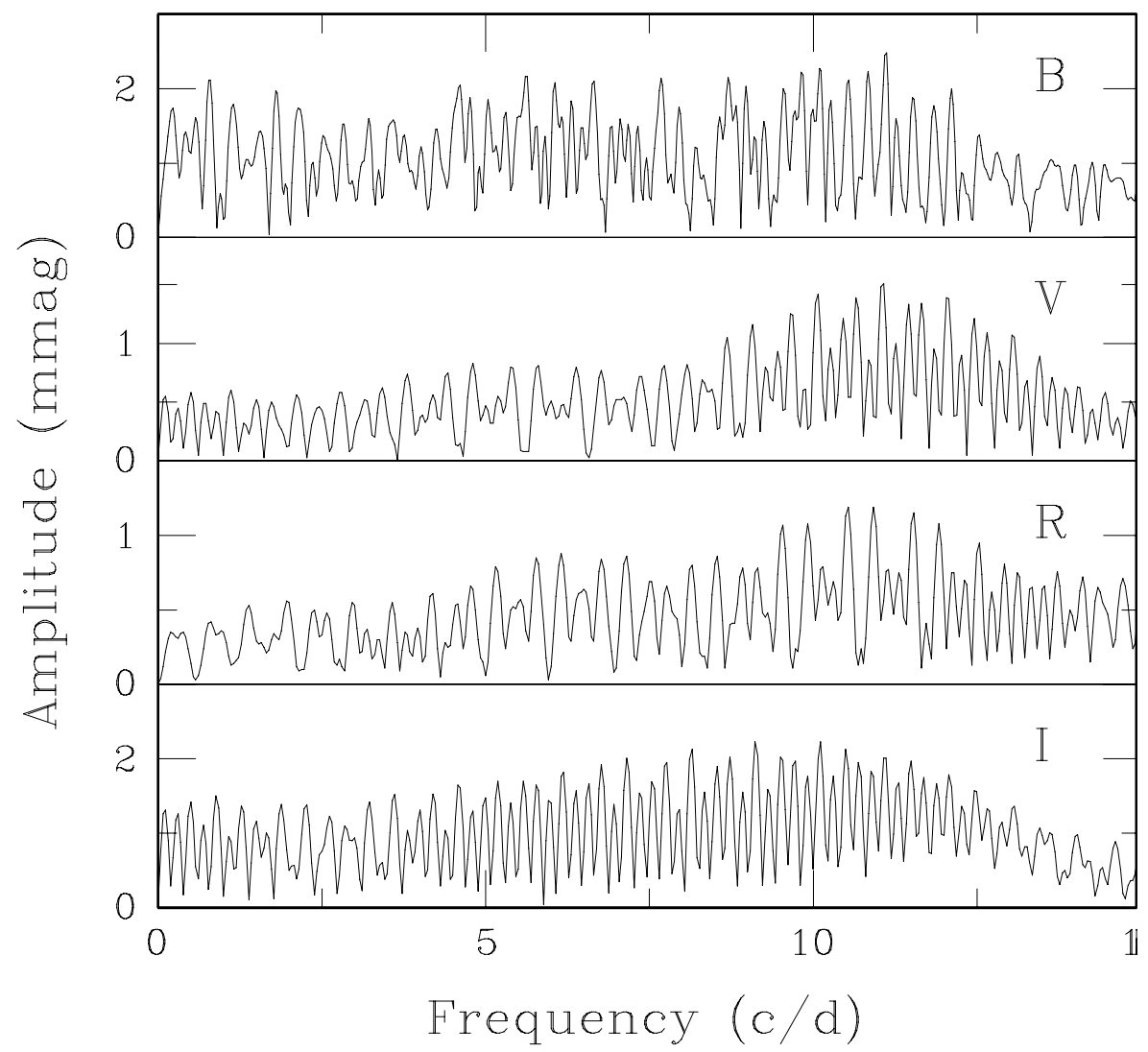

Figure 8. As for Fig. 7, but after pre-whitening by another one $(I)$ or two $(B V R)$ frequencies. Note the different vertical scales of the different panels. 


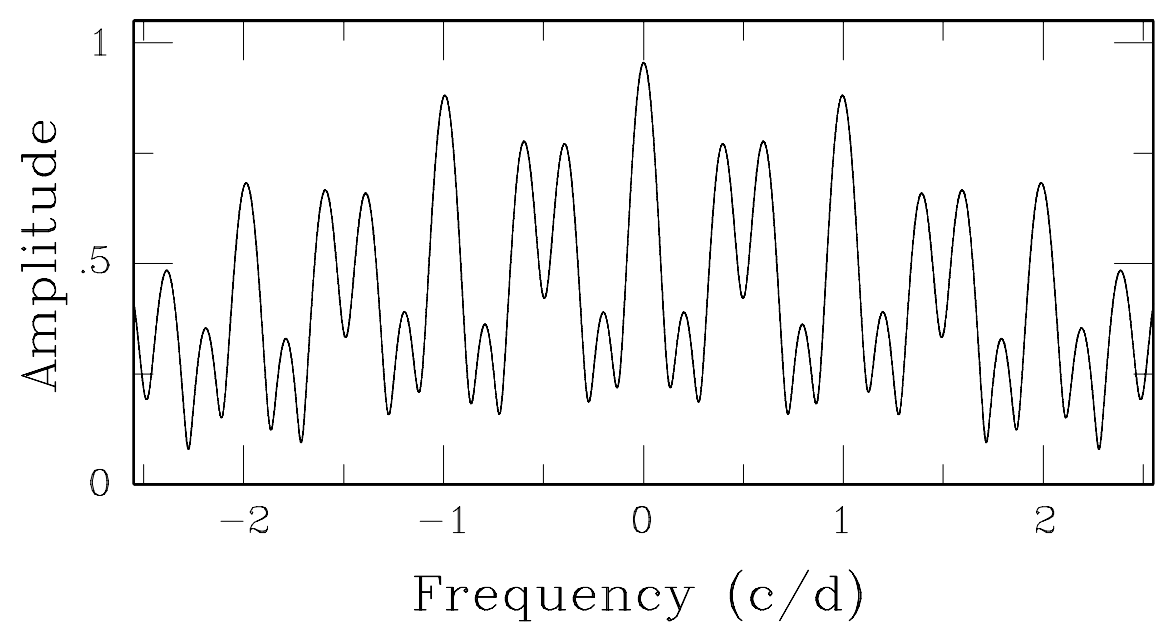

Figure 9. The window function of the SAAO $R$-filter observations. Window functions for the $V$ and $I$ filters are virtually identical.

Table 5. The results of fitting the frequencies 5.05336 and $3.62813 \mathrm{~d}^{-1}$, and their first harmonics, to the SAAO data. For each frequency, the first line gives amplitudes and the second line gives phases. The uncertainty in the last digit of an entry is given in brackets.

\begin{tabular}{ccccc}
\hline Frequency $\left(\mathrm{d}^{-1}\right)$ & \multicolumn{4}{c}{ Amplitude (mmag)/phase (rad) } \\
& $B$ & $V$ & $R$ & $I$ \\
\hline \multirow{2}{*}{5.05336} & $41.7(3)$ & $41.1(5)$ & $35.8(5)$ & $27.9(6)$ \\
& $2.875(8)$ & $2.93(1)$ & $2.91(1)$ & $2.88(2)$ \\
3.62813 & $15.7(3)$ & $15.6(5)$ & $14.4(6)$ & $13.0(6)$ \\
& $0.52(2)$ & $0.95(3)$ & $0.93(4)$ & $0.92(5)$ \\
7.25625 & $6.6(3)$ & $6.5(4)$ & $6.2(4)$ & $4.7(5)$ \\
& $-0.41(4)$ & $-0.22(6)$ & $-0.27(6)$ & $-0.2(1)$ \\
10.10672 & $1.6(3)$ & $1.6(4)$ & $1.0(4)$ & $2.1(5)$ \\
& $1.3(2)$ & $0.7(2)$ & $0.6(4)$ & $-0.2(2)$ \\
\hline
\end{tabular}

Table 6. The spectroscopic observing log: $T_{\text {exp }}$ is the exposure time.

\begin{tabular}{cccc}
\hline $\begin{array}{c}\text { Start exposure time } \\
(\text { HJD 2455120+) }\end{array}$ & $\begin{array}{c}T_{\text {exp }} \\
(\mathrm{s})\end{array}$ & $\begin{array}{c}\text { Resolution } \\
(\AA)\end{array}$ & $\begin{array}{c}\text { Usable wavelength range } \\
(\AA)\end{array}$ \\
\hline 3.6116 & 900 & 2 & $3700-5400$ \\
4.6239 & 600 & 1 & $4000-4750$ \\
\hline
\end{tabular}

young, if it were indeed rapidly rotating. A further question is whether both $P_{1}$ and $P_{2}$ could be ascribed to rotation, that is, whether differential rotation with $\Delta P=0.078 \mathrm{~d}$ is possible. Barnes et al. (2005) studied differential rotation in 10 stars with late spectral types (G-M2); in particular, they postulated the rotation law

$\Omega(\theta)=\Omega_{\mathrm{e}}-\Delta \Omega \sin ^{2} \theta$,

where $\theta$ is the latitude on the stellar surface and $\Omega_{\mathrm{e}}$ is the equatorial rotation rate. For present purposes, fig. 3 of Barnes et al. (2005) is particularly relevant: it shows a plot of observed $\log (\Delta \Omega)$ against $\log (\Omega)$, together with a linear fit to the data. At a rotation rate of $\Omega=2 \pi / P_{1}=22.80 \mathrm{rad} \mathrm{d}^{-1}$, the fitted line predicts $\Delta \Omega \approx 0.079$. Using the approximation

$\frac{\Delta P}{P} \approx \frac{\Delta \Omega}{\Omega}$,

$\Delta P \approx 0.0035$, more than 20 times smaller than the required period difference. There is considerable scatter in the $\log \Delta \Omega-\log \Omega$ plot, but even using the largest observed value $\Delta \Omega \sim 1$ does not give $\Delta P$ large enough.
The two higher frequencies were ignored in the discussion of rotation. It seems highly improbable that four independent frequencies could be accounted for by four groups of spots at four different latitudes, not to mention the extreme rotation rate, which would be required to explain the $10.11 \mathrm{~d}^{-1}(P=0.099 \mathrm{~d}$ or $2.37 \mathrm{~h}$ ) variation. This means that spot configurations, which give rise to nonsinusoidal flux modulation, need to be invoked. Although this may be possible, the author is not aware of any observations in support of such configurations.

The material above can be summarized by saying that one periodicity may be due to rotation, but the remainder must be caused by pulsation. In other words, pulsation has to play a role. Had the star been a giant, then pulsation is the only physically possible explanation of the periodicities.

There are some apparent problems with V760 being a pulsator, most notably that it does not lie in the classical instability strip. Very little theoretical work seems to have been done on pulsation in stars at the bottom of the main sequence. One exception is the study by Gabriel \& Grossman (1977) of the stability against radial pulsations of main-sequence stars with $M \leq 0.5 \mathrm{M}_{\odot}$. Of particular interest is the periods of the fundamental modes, namely 30-44 min for star with $0.3 \leq M \leq 0.5 \mathrm{M}_{\odot}$. This indicates that the periods expected in slightly more massive $\mathrm{K}$ dwarfs should be too short to explain the observations of V760. Furthermore, as far as the author is aware, pulsations have not previously been detected in main-sequence $\mathrm{K}$ or M stars.

On the other hand, periodic variability, almost certainly due to pulsation, has been found in $\mathrm{K}$ giants, mostly from radial velocity measurements (e.g. Hatzes, Döllinger \& Endl 2007). Some of the periods found have been quite short - a few hours. Brightness variations have also been discovered photometrically, primarily with periods longer than a day. An exception is the low-amplitude, shortperiod variability discovered in a K giant by Kallinger et al. (2005). It appears to be generally accepted that the periodicities for $\mathrm{K}$ giants are due to radial pulsations.

If V760 is pulsating with periods 0.198 and $0.276 \mathrm{~d}$, and the remaining two frequencies are harmonics, then the pulsations strongly resemble the double-mode pulsations in Cepheids and RR Lyrae stars, as is evident from the following points:

(i) The ratio of the shorter to longer period is 0.71 , very similar to the ratio of fundamental to first overtone periods in Cepheids and RR Lyrae stars. 


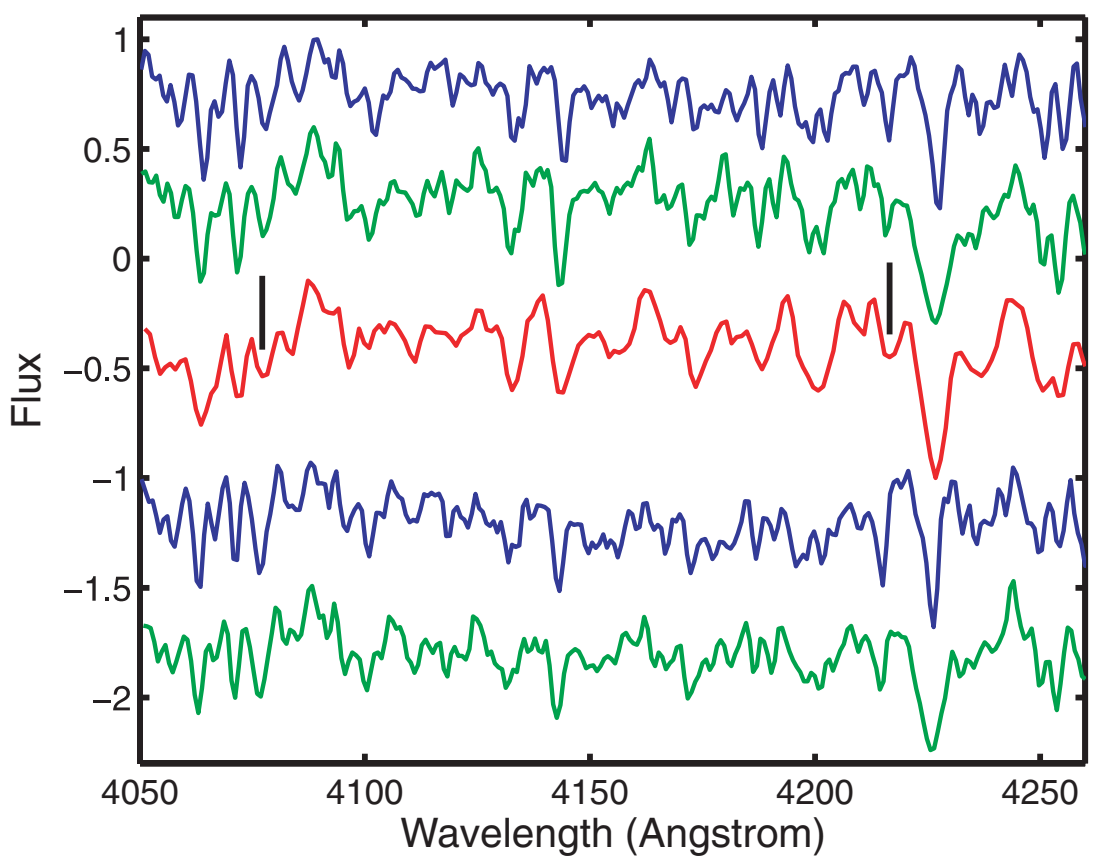

Figure 10. A comparison of a section of a 2- $\AA$ spectrum of V760 with those of MK standards. From top to bottom: K0V and K5V standards; V760; K0III and K5III standards. The flux zero-points are arbitrary. The short vertical bars mark the positions of the Sr I 4077 and Sr II $4215 \AA$ absorption features in the V760 spectrum.

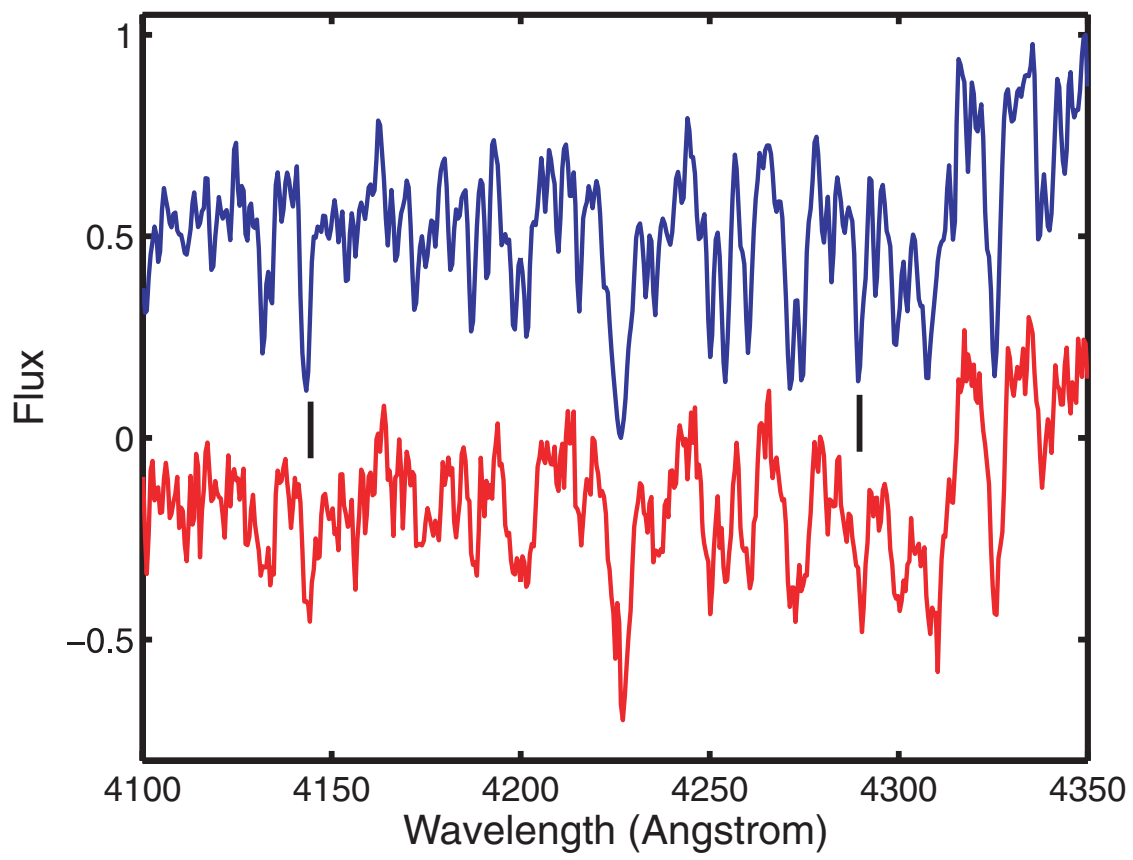

Figure 11. A comparison of part of a 1- $\AA$ spectrum of V760 (bottom) with that of the bright K4/5V star, HR 8387, observed on the same night, with exactly the same instrumental setup. The short vertical bars mark the positions of the Fe I and Cr I absorption lines at 4144 and $4290 \AA$, respectively.

(ii) The pulsations with the longer period are distinctly nonsinusoidal, while those with the shorter period are close to sinusoidal. The light curve shape of the lower frequency variation in V760 (Fig. 12) is also very typical of fundamental mode light curves for Cepheids and RR Lyrae stars.

(iii) In the RR Lyrae stars, the amplitude of the higher frequency mode (overtone) is always larger than that of the fundamental mode (e.g. Gautschy \& Saio 1996): the same is true for V760.
If $\mathrm{V} 760$ is in fact pulsating in a radial mode, then it may lie on the extension of a known period-luminosity (PL) relation to very short periods. Sandage \& Tammann (2006) provide a review of various PL relations. From the relations for Population II Cepheids, and 'above horizontal branch' stars, $M_{V}=0.87$ and 1.02 are obtained for $P=P_{1}=0.276 \mathrm{~d}$. These absolute magnitudes are not very different from $M_{V}=0.81$ from the Cepheid PL relation. This is a little faint for a K giant (e.g. Schmidt-Kaler 1982), although not 


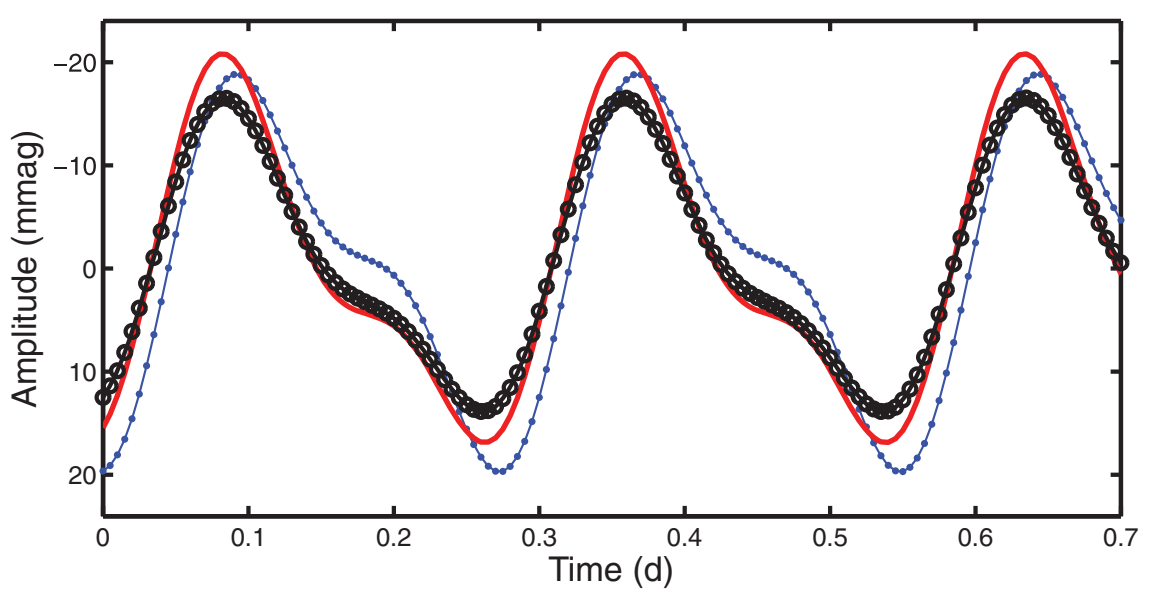

Figure 12. The shape of the non-sinusoidal variation with $f=3.628 \mathrm{~d}^{-1}$. Dot-dashed line $-B$; solid line $-V$; open circles $-I$. The variation in $R$ is intermediate between that in $V$ and $I$, and is not plotted in order to have a less-cluttered diagram.

excessively so, but far too bright for a dwarf $\operatorname{star}\left(M_{V}>6\right.$ for a K2V star, e.g. Schmidt-Kaler 1982; Bilir et al. 2008).

Of course, if $P=0.276 \mathrm{~d}$ is the fundamental mode period, then this implies restrictions on the physical properties of the star. The pulsation constant $Q$ is defined by

$P \sqrt{\bar{\rho}}=Q$.

The equation can be rewritten in the form

$\log Q=-6.456+\frac{1}{2} \log g+0.1 M_{\mathrm{bol}}+\log T_{\text {eff }}-\log f$

(e.g. Breger 2000). Using $M_{\text {bol }}=6.0$ and $\log T_{\text {eff }}=3.69$ for a K2V star (Schmidt-Kaler 1982),

$\log Q=-2.73+\frac{1}{2} \log g$

or

$\log g=5.46+2 \log Q$

for $f=3.628 \mathrm{~d}^{-1}$. For the fundamental mode, it is usually assumed that $0.03 \lesssim Q \lesssim 0.12$; this implies $2.4 \lesssim \log g \lesssim 3.6$, values which are low for a dwarf $(\log g \sim 4.5)$ and high for a giant $(\log g \sim 2)$.

An alternative form for the pulsation equation (3) is

$\log Q=-12.7085+\frac{1}{2} \log \frac{M}{\mathrm{M}_{\odot}}+0.3 M_{\text {bol }}+3 \log T_{\text {eff }}-\log f$

(Breger 2000). For the same parameter values as before,

$M / \mathrm{M}_{\odot}=6.3 Q^{2}$

follows and if $0.03<Q<0.12$, then $0.006<M<0.09 \mathrm{M}_{\odot}$, which is of course far too low for the spectral type.

The problem would be exacerbated if the pulsations were overtones. In that case, the pulsation constant $Q$ would be smaller and hence the mass derived from (5) even smaller. On the other hand, if V760 were a giant, then the pulsation periods could possibly be consistent with overtone pulsations, as the following discussion shows. Observationally, a number of double-mode Cepheids and RR Lyrae have been found to pulsate in the first and second overtone modes (e.g. Soszyński et al. 2008a, Olech \& Moskalik 2009). However, the typical period ratios are about 0.8 . By contrast, Kallinger et al. (2005) present theoretical models for pulsation in a K2.5 giant in which the ratio of second overtone to first overtone period is about 0.7 (their fig. 11). Interestingly, the two frequencies
Table 7. Fourier coefficients calculated from the UNSW data $\left(5.053+10.107 \mathrm{~d}^{-1}\right.$ frequencies) and from the SAAO data $\left(3.628+7.256 \mathrm{~d}^{-1}\right.$ frequencies).

\begin{tabular}{cccc}
\hline Frequencies $\left(\mathrm{d}^{-1}\right)$ & Filter & $R_{21}$ & $\phi_{21}$ \\
\hline $5.053+10.107$ & $I_{\mathrm{J}}$ & 0.13 & 5.76 \\
$3.628+7.256$ & $B$ & 0.42 & 4.83 \\
& $V$ & 0.41 & 4.17 \\
& $R$ & 0.43 & 4.15 \\
& $I_{\mathrm{C}}$ & 0.37 & 4.25 \\
\hline
\end{tabular}

involved are $\sim 4.5-5$ and $\sim 3.4-3.5 \mathrm{~d}^{-1}$, for stellar masses in the range $1.2-1.6 \mathrm{M}_{\odot}$.

Table 7 contains the Fourier coefficients derived from Table 5. Also shown are the coefficients determined from fitting $f=$ $5.05336 \mathrm{~d}^{-1}$, and its first harmonic, to the UNSW data. Unfortunately, a comparison to $\log P-R_{21}$ and $\log P-\phi_{21}$ diagrams, such as those in Soszyński et al. (2008a,b), for Cepheids and W Vir stars, respectively, does not give a clear-cut indication of possible overtone numbers.

It should also be pointed out that the observed $\mathrm{K}$ giant pulsations referred to above are usually interpreted as similar to solar oscillations (e.g. De Ridder et al. 2009). Solar-like oscillations are stochastically excited, that is, the modes have short lifetimes (typically a few days). The pulsation frequencies of V760 lie well within the range of values recently observed in K giants by COROT (Hekker et al. 2009), but the V760 modes are evidently long-lived. In this regard, the discovery of long-lived modes in some $\mathrm{K}$ giants (De Ridder et al. 2009) is quite interesting. It should be noted though that the amplitudes seen in V760 are two orders of magnitudes larger than those detected in pulsating $\mathrm{K}$ giants (e.g. Kallinger et al. 2005).

\section{CONCLUSIONS}

It was shown above that at least one of the two lower frequencies observed in V760 has to be due to pulsations. Arguments in favour of both periodicities being caused by pulsations are (i) their distinct non-sinusoidal nature and (ii) the period ratio of 0.71 , often seen in pulsating stars. Ellipsoidal variability, or a star-spot, could, in principle, be responsible for one of the periodicities, but it seems unlikely. 
A thorough study of V760 is certainly merited. Spectroscopically, it is a $\mathrm{K}$ dwarf star, but appears to show pulsation behaviour, which is more characteristic of a giant. If it is indeed a dwarf, it is, to the best of the author's knowledge, the only pulsating $\mathrm{K}$ dwarf, which has been identified as such.

With the advent of the intensive monitoring of large areas of sky, as required by, for examples, microlensing and exoplanet studies, literally tens of thousands of new pulsating variable stars have been found. Classification of new variables is, of course, conditioned by what has gone before, and it is quite conceivable that new types of variables will be erroneously classified under existing headings. None the less, it seems remarkable that variables similar to V760, with its fairly large amplitude and short periods, have been overlooked - there are no overt selection effects at work. A plausible explanation is that such variables are intrinsically rare. This could be the case if variables like V760 are in a relatively short-lived evolutionary state.

The X-rays, and emission lines, indicate that V760 is probably a young star. The star is within a few degrees of the young (5$8 \mathrm{Myr}) \eta$ Chamaeleontis cluster and has similar spatial motion to the cluster members (Song, Zuckerman \& Bessell 2004, and below). However, the cluster is very compact (less than a degree across, on the sky - e.g. Luhman 2004) and V760 appears too bright for its $\left(I-K_{\mathrm{S}}\right)$ colour in a $K_{\mathrm{S}}-\left(I-K_{\mathrm{S}}\right)$ plot for the cluster members (Song et al. 2004, fig. 1). If the (unknown) absorption is neglected, then the ratio of X-ray to bolometric luminosity of V760 can be derived. This is most easily done by scaling the observations of HD 98800 presented by Kastner et al. (1997). The ROSAT measurement of V760 was 0.0337 counts s$^{-1}$ and for HD 98800, it was 0.66 counts $\mathrm{s}^{-1}$. Apparent magnitudes of the stars are $V=12.3$ (V760) and $V=8.9$ (HD 98800); bolometric corrections are -0.4 (V760, assuming a spectral type K2) and -0.7 (HD 98800, spectral type K5). For HD 98800, $\log \left(L_{\mathrm{X}} / L_{\mathrm{bol}}\right)=-3.4$ and it follows that for V760, $\log \left(L_{\mathrm{X}} / L_{\mathrm{bol}}\right) \approx-3.2$. In a $\log \left(L_{\mathrm{X}} / L_{\mathrm{bol}}\right)-(B-V)$ plot, this places V760 amongst members of the Pleiades (age $\sim 10^{8} \mathrm{yr}$ ) and young (age < $100 \mathrm{Myr}$ ) nearby stars (Zuckerman \& Song 2004, fig. 4).

Use can also be made of the V760 X-ray hardness ratios: these are HR $1=-0.1$ and HR2 $=-1.0$ with errors of 0.32 and 0.39 , respectively (Voges et al. 2000). Kastner et al. (2003) show HR1-HR2 plots for a number of young associations. The V760 hardness ratio HR2 is particularly soft when compared to values for very young objects, but the errors are so large that virtually any interpretation is possible.

The kinematic implications of the star having an absolute magnitude $M_{V} \sim 0.5-1$, as predicted by the PL relations, are interesting. Proper motion component measurements in the recent literature are $\mu_{\alpha}=-7.3, \mu_{\delta}=18.3$ (NOMAD catalogue, Zacharias et al. 2004); $\mu_{\alpha}=-9.6, \mu_{\delta}=16.3$ (UCAC3 catalogue, Zacharias et al. 2009); and $\mu_{\alpha} \cos \delta=-9.6, \mu_{\delta}=15.9$ mas yr $^{-1}$ (PPMX catalogue, Röser

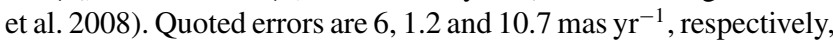
for the three catalogues. The total proper motion follows from

$\mu=\sqrt{\mu_{\alpha}^{2} \cos ^{2} \delta+\mu_{\delta}^{2}}$.

The standard error on $\mu$ can be approximated using the delta method, neglecting any correlation between measurements of $\mu_{\alpha}$ and $\mu_{\delta}$, as

s.e. $(\mu) \approx \frac{1}{\mu}\left[\mu_{\alpha}^{2} \cos ^{4} \delta \operatorname{var}\left(\mu_{\alpha}\right)+\mu_{\delta}^{2} \operatorname{var}\left(\mu_{\delta}\right)\right]^{1 / 2}$

for the NOMAD and UCAC3 catalogue values and

s.e. $(\mu) \approx \frac{1}{\mu}\left[\left(\mu_{\alpha} \cos \delta\right)^{2} \operatorname{var}\left(\mu_{\alpha} \cos \delta\right)+\mu_{\delta}^{2} \operatorname{var}\left(\mu_{\delta}\right)\right]^{1 / 2}$ for the PPMX measurements. The total proper motions derived from the values given in the three catalogues are then 18.5 (5.9),

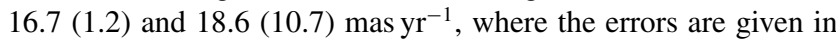
brackets. The values agree quite well and we proceed to use the UCAC3 result below, because it has the smallest error.

The transverse velocity can be calculated from

$v_{\mathrm{T}}=4.74 \mu D$,

where $\mu$ is in $\operatorname{arcsec} \mathrm{yr}^{-1}$, and the distance, derived from

$\log D=1+\frac{1}{5}\left(m_{V}-M_{V}\right)$

is in pc. A formula for the error on $v_{\mathrm{T}}$ is obtainable from the delta method:

s.e. $\left(v_{\mathrm{T}}\right) \approx v_{\mathrm{T}}\left[\frac{\operatorname{var}(\mu)}{\mu^{2}}+\frac{\operatorname{var}(D)}{D^{2}}\right]^{1 / 2}$

or, if the uncertainty in $m_{V}$ is neglected,

s.e. $\left(v_{\mathrm{T}}\right) \approx v_{\mathrm{T}}\left[\frac{\operatorname{var}(\mu)}{\mu^{2}}+0.2121 \operatorname{var}\left(M_{V}\right)\right]^{1 / 2}$.

The apparent magnitude of V760 is $m_{V}=12.30$, and if it is assumed that $M_{V}$ is in the range $0.5-1$, a distance $1820-2290$ pc follows. With $\mu=16.7$, the transverse velocity is in the range $144-181 \mathrm{~km} \mathrm{~s}^{-1}$. From equation (6), the uncertainty in the velocity due to the proper motion error alone is only 7.2 per cent. Of course, the true error is bound to be higher due to the contribution from the unknown measurement error in $M_{V}$

Such high values of $v_{\mathrm{T}}$ are usually associated with Population II stars. The Galactic latitude of V760 is $b=-19.726$; hence, its distance from the Galactic plane would be $614-773$ pc (using the range of values of $D$ found above). This value may be compared to the criterion $|z|>600 \mathrm{pc}$ used by Harris (1985) to identify Type II Cepheid (W Vir) stars.

It is noteworthy that no double-mode W Vir stars have been discovered (e.g. Percy 2007). It must also be said that the spectra of V760 show no overt signs of metal-deficiency.

An obvious next step (at least observationally) is to obtain time series spectroscopy of V760. If the interpretation of the photometry is correct, then this should show low-amplitude radial velocity variations with periods $P_{1}$ and $P_{2}$. It may also lead to the discovery of further pulsations and help with the identification of the modes involved. It will be useful to obtain a red spectrum of V760 in order to check for the presence of Li absorption, in order to place limits on the age of the star.

\section{ACKNOWLEDGMENTS}

The author is grateful for telescope time made available at the SAAO and for the support of the SAAO technical staff. David Kilkenny (Dept. Physics, University of the Western Cape) kindly obtained spectra of UNSW-V-760 and Richard Gray (Dept. Physics, Appalachian State University) generously discussed the classification with the author. Francois van Wyk (SAAO) is thanked for his $0.5-\mathrm{m}$ photometry of V760.

\section{REFERENCES}

Barnes J. R., Collier Cameron A., Donati J.-F., James D. J., Marsden S. C., Petit P., 2005, MNRAS, 357, L1

Bessell M. S., 1979, PASP, 91, 589

Bessell M. S., Brett J. M., 1988, PASP, 100, 1134

Bessell M. S., Castelli F., Plez B., 1998, A\&A, 333, 231 
Bilir S., Karaali S., Ak S., Yaz E., Cabrera-Lavers A., Coşkunoğlu K. B., 2008, MNRAS, 390, 1569

Breger M., 2000, in Breger M., Montgomery M. H., eds, ASP Conf. Ser. Vol. 210, Delta Scuti and Related Stars: Reference Handbook and Proceedings of the 6th Vienna Workshop in Astrophysics. Astron. Soc. Pac., San Francisco, p. 3

Christiansen J. L. et al., 2008, MNRAS, 385, 1749

De Ridder J. et al., 2009, Nat, 459, 398

Epchtein N. et al., 1999, A\&A, 349, 236

Fouqué P. et al., 2000, A\&AS, 141, 313

Gabriel M., Grossman A. S., 1977, A\&A, 54, 283

Gautschy A., Saio H., 1996, ARA\&A, 34, 551

Gray R. O., Corbally C. J., 2009, Stellar Spectral Classification, Princeton Univ. Press, Princeton, NJ

Harris H. C., 1985, AJ, 90, 756

Hatzes A. P., Döllinger M. P., Endl M., 2007, Comm. Asteroseismol., 150, 115

Hekker S. et al., 2009, A\&A, 506, 465

Kallinger Th., Zwintz K., Pamyatnykh A. A., Guenther D. B., Weiss W. W., 2005, A\&A, 433, 267

Kastner J. H., Zuckerman B., Weintraub D. A., Forveille T., 1997, Sci, 277, 67

Kastner J. H., Crigger L., Rich M., Weintraub D. A., 2003, ApJ, 585, 878

Koen C., Marang F., Kilkenny D., Jacobs C., 2007, MNRAS, 380, 1433

Luhman K. L., 2004, ApJ, 616, 1033
Meibom S., Mathieu R. D., Stassun K. G., 2009, ApJ, 695, 679

Menzies J. W., Cousins A. W. J., Banfield R. M., Laing J. D., 1989, SAAO Circ., 13, 1

Moran E. C., Helfand D. J., Becker R. H., White R. L., 1996, ApJ, 461, 127

Olech A., Moskalik P., 2009, A\&A, 494, L17

Percy J. R., 2007, Understanding Variable Stars. Cambridge Univ. Press, Cambridge

Röser S., Schilbach E., Schwan H., Kharchenko N. V., Piskuniv A. E., Scholz R.-D., 2008, A\&A, 488, 401

Sandage A., Tammann G. A., 2006, ARA\&A, 44, 93

Schechter P. L., Mateo M., Saha A., 1993, PASP, 105, 1342

Schmidt-Kaler T., 1982, in Schaifers K., Voigt H. H., eds, Landolt-Börnstein Series, Vol. 2b. Springer-Verlag, Berlin, p. 17

Skrutskie M. F. et al., 2006, AJ, 131, 1163

Song I., Zuckerman B., Bessell M. S., 2004, ApJ, 600, 1016

Soszyński I. et al., 2008a, Acta Astron., 58, 163

Soszyński I. et al., 2008b, Acta Astron., 58, 293

Voges W. et al., 2000, IAUC, 7432, 1

Wade R. A., Hoessel J. G., Elias J. H., Huchra J. P., 1979, PASP, 91, 35

Zacharias N., Monet D. G., Levine S. E., Urban S. E., Gaume R., Wycoff G. L., 2004, BAAS, 205, 4815 (Vizier yCat 1297)

Zacharias N. et al., 2009, Vizier yCat 1315

Zuckerman B., Song I., 2004, ARA\&A, 42, 685

This paper has been typeset from a $\mathrm{T}_{\mathrm{E}} \mathrm{X} / \mathrm{L} \mathrm{T} \mathrm{E} \mathrm{X}$ file prepared by the author. 